\title{
Comunidade de liquens corticícolas em um gradiente de urbanização na Bacia Hidrográfica do Rio dos Sinos, no sul do Brasil
}

\author{
Corticolous lichen community in an urbanization gradient \\ in the Rio dos Sinos Hydrographic Basin, southern Brazil
}

\author{
Fabiane Lucheta ${ }^{1,4}$, Natália Mossmann Koch $^{2}$, Suzana Maria de Azevedo Martins ${ }^{3}$ \& Jairo Lizandro Schmitt ${ }^{1}$
}

\begin{abstract}
Resumo
Liquens são mundialmente conhecidos como indicadores de qualidade ambiental porque são sensíveis às modificações do ambiente, especialmente as de origem antrópica. Neste estudo foram avaliadas a riqueza, a composição e a cobertura de comunidades de liquens corticícolas em nove municípios com diferentes graus de urbanização e verificadas as principais semelhanças na composição das espécies entre as comunidades. Os locais amostrados estavam distribuídos em uma matriz rural, rural/urbana ou urbana/ industrial. Dez forófitos foram amostrados em cada área, utilizando o método do elástico. Ao total, foram identificadas 215 espécies de liquens, das quais nove são novas ocorrências, sendo quatro para o Brasil e cinco para o Rio Grande do Sul. Com o aumento da urbanização, observou-se uma simplificação da estrutura da comunidade de liquens, com diminuição da riqueza e mudanças na composição das espécies. Além disso, na matriz urbana/industrial poucas espécies apresentaram alta porcentagem de cobertura e, em conjunto, somaram grande importância na comunidade. Maior homogeneidade da composição de espécies foi encontrada em áreas mais próximas e inseridas na matriz urbano/industrial. Este estudo demonstrou que a arborização urbana é um fator importante para o estabelecimento das espécies de liquens, ao longo de um gradiente de urbanização.
\end{abstract}

Palavras-chave: cobertura, composição, gradiente rural-urbano, riqueza de espécies.

\begin{abstract}
Lichens are known worldwide as indicators of environmental quality because they are sensitive to environmental changes, specially the ones with anthropogenic origin. In this study, we analyzed species composition, richness and coverage of corticolous lichen communities in nine municipalities with different urbanization levels, and evaluated their similarities considering lichen species composition. The studied sites were distributed in rural, rural/urban and urban/industrial matrices. Ten phorophytes were sampled in each area using the rubber band method. A total of 215 lichen species were identified, of which nine species are new records: four are being cited for Brazil for the first time, and five for Rio Grande do Sul. As urbanization increased, a simplification of the lichen community structure was observed, as well as a decrease in species richness and changes on species composition. Besides, in the urban/industrial matrix, few species had high coverage percentage and together added great importance to the community. We found higher homogeneity on species composition in closer areas and in the ones that were inserted in the urban/industrial matrix. This study showed that urban tree vegetation is an important factor for lichen species establishment along an urbanization gradient.
\end{abstract}

Key words: coverage, composition, rural-urban gradient, species richness.

Veja material suplementar em <https://figshare.com/s/7552d3a29e032053914a>

\footnotetext{
${ }^{1}$ Universidade Feevale, Prog. Pós-graduação em Qualidade Ambiental, Lab. Botânica, Rod. ERS-239 2755, 93525-075, Novo Hamburgo, RS, Brasil.

${ }^{2}$ Universidade Federal de Mato Grosso do Sul, Prog. Pós-graduação em Biologia Vegetal, Av. Costa e Silva s/n, 79070-900, Campo Grande, MS, Brasil.

${ }^{3}$ Fundação Zoobotânica do Rio Grande do Sul, Museu de Ciências Naturais, R. Dr. Salvador França 1427, 90690-000, Porto Alegre, RS, Brasil.

${ }^{4}$ Autor para correspondencia: fabiane.lucheta@gmail.com
} 


\section{Introdução}

Liquens são organismos simbióticos compostos por um ou mais fungos (micobionte) e uma ou mais algas verdes ou cianobactérias (fotobionte) (Kricke \& Loppi 2002; Spribille et al. 2016). Como resultado desta associação entre os simbiontes, eles apresentam um talo morfologicamente distinto que pode ser classificado como crostoso, folioso, fruticoso, filamentoso, esquamuloso ou dimórfico (Nash III 2008). Os liquens são organismos perenes que mantém a uniformidade ao longo do tempo e que apresentam ampla distribuição geográfica, podendo ser encontrados em vários substratos como no córtex de árvores (corticícolas), em rochas (saxícolas), no solo (terrícolas) e nas folhas (folícolas) (Brodo et al. 2001).

Liquens são amplamente estudados como indicadores biológicos, sendo que a avaliação da qualidade ambiental em uma área específica pode ser feita por meio do mapeamento das suas espécies (Conti \& Cecchetti 2001). A frequência, a riqueza, a cobertura e a composição de espécies de liquens em uma porção definida do tronco das árvores podem ser utilizadas como estimativas de diversidade, de parâmetros para avaliar o grau de estresse ambiental e para definir a qualidade de um determinado local (Asta et al. 2002; Käffer et al. 2012).

No Brasil, ainda poucos são os estudos que avaliaram a estrutura da comunidade de liquens (Marcelli 1992; Cáceres et al. 2008), sendo a maioria deles realizados no Rio Grande do Sul, em áreas de Mata Atlântica, tal como o de Käffer et al. (2010) e de Koch et al. (2012) que inventariaram 113 e 78 espécies, respectivamente. Em restinga, Martins \& Marcelli (2011) registraram 126 espécies de liquens crescendo sobre 30 forófitos. Na região do Bioma Pampa, Käffer et al. (2015) inventariam 254 espécies em 60 forófitos.

A urbanização altera o ambiente natural, provocando impactos ambientais como mudanças climáticas, poluição atmosférica e perda da biodiversidade (McKinney 2006; Lättman et al. 2014; Munzi et al. 2014). Estudos têm demonstrado alteração na composição e diminuição gradual da riqueza de espécies de epífitos vasculares (Becker et al. 2017), de besouros (Niemelä \& Kotze 2009), de aves (Gagne \& Fahrig 2011) e de liquens (Washburn \& Culley 2006; Koch et al. 2016) no sentido área rural-urbana.
Em área urbana no Rio Grande do Sul, Käffer et al. (2011) avaliaram a comunidade de liquens em 33 bairros da cidade de Porto Alegre e uma área de referência na cidade de Viamão, encontrando 144 táxons e vários novos registros de espécies para o estado. Koch et al. (2016) estudaram a comunidade de liquens em sete municípios com diferentes graus de urbanização e industrialização, registrando 225 espécies de liquens, das quais a riqueza por unidade amostral foi de 42 espécies em Montenegro, e a mínima, em Triunfo, de oito espécies.

Este estudo teve como objetivos: 1) avaliar a riqueza, a composição e a cobertura de espécies de liquens corticícolas em nove municípios da Bacia Hidrográfica do Rio dos Sinos, que apresentam diferentes graus de urbanização; 2) verificar as principais semelhanças na composição das espécies entre as comunidades inventariadas.

\section{Material e Métodos}

Área de estudo

A Bacia Hidrográfica do Rio dos Sinos (BHRS) está situada a nordeste do Rio Grande do Sul e possui uma área de $3.820 \mathrm{~km}^{2}$, correspondendo a $1,5 \%$ da área total desse estado. A população aproximada da BHRS é de 1.249.100 habitantes, sendo que $90,6 \%$ deles ocupam as áreas urbanas (FEPAM 2016; SEMA 2016). Ela é uma das mais importantes bacias hidrográficas do estado, compreendendo 32 municípios (Figueiredo et al. 2010) com diferentes graus de urbanização.

$\mathrm{O}$ estudo foi realizado em nove municípios localizados ao longo da BHRS, sendo eles: Caraá, Rolante, Santo Antônio da Patrulha, Parobé, Sapiranga, Estância Velha, Novo Hamburgo, São Leopoldo e Canoas. Os nove municípios foram classificados conforme sua matriz em um gradiente de urbanização, baseado no percentual de área urbana em um raio de $3.200 \mathrm{~m}$ no entorno de cada área amostral, como rural (0-25\%), rural/urbano (25-50\%) e urbano/industrial (50-100\%). Esse percentual foi obtido a partir do mapa de cobertura vegetal do Rio Grande do Sul, ano base 2002 (Hasenack et al., comunicação pessoal), utilizando o software ArcMap (Tab. 1; Fig. 1).

Os municípios selecionados estão no máximo $25 \mathrm{~km}$ distantes um do outro, apresentam clima Cfa (subtropical úmido) (Peel et al. 2007). Eles estão inseridos na formação de Floresta Estacional Semidecidual do Bioma Mata Atlântica (Anschau 2016) e a altitude varia de 15 a $52 \mathrm{~m}$ (Tab. 1). 
Tabela 1 - Localização geográfica, matriz principal, altitude, densidade demográfica, área e percentual de área urbana no entorno de cada município estudado da BHRS, RS, Brasil.

Table 1 - Geographic location, main matrix, altitude, demographic density, area and percentage of urban area around each municipality of the BHRS, RS, Brazil.

\begin{tabular}{|c|c|c|c|c|c|c|c|}
\hline Município & Sigla & $\begin{array}{l}\text { Localização } \\
\text { Geográfica }\end{array}$ & Matriz & $\begin{array}{l}\text { Altitude } \\
\text { (m) }\end{array}$ & $\begin{array}{c}\text { Densidade } \\
\text { Demográfica } \\
\left(\mathbf{H a b} / \mathbf{k m}^{2}\right)\end{array}$ & $\begin{array}{l}\text { Área } \\
\left(\mathbf{K m}^{2}\right)\end{array}$ & $\begin{array}{c}\text { Área } \\
\text { Urbana } \\
(\%)\end{array}$ \\
\hline Caraá & $\mathrm{CA}$ & $\begin{array}{c}29^{\circ} 47^{\prime} 4,85^{\prime \prime} \mathrm{S} \\
50^{\circ} 25^{\prime} 39,80 \text { "O }\end{array}$ & & 44 & 24,84 & 294,5 & 0 \\
\hline Santo Antônio da Patrulha & ST & $\begin{array}{c}29^{\circ} 49^{\prime} 13,76 ” \mathrm{~S} \\
50^{\circ} 31^{\prime} 5,08^{\prime \prime} \mathrm{O}\end{array}$ & Rural & 47 & 37,8 & 1049,8 & 22 \\
\hline Rolante & RO & $\begin{array}{c}29^{\circ} 39^{\prime} 5,87^{\prime \prime} \mathrm{S} \\
50^{\circ} 34^{\prime} 33,75 \text { "O }\end{array}$ & & 45 & 65,91 & 295,6 & 12 \\
\hline Parobé & PA & $\begin{array}{c}29^{\circ} 37^{\prime} 47,80^{\prime \prime} \mathrm{S} \\
50^{\circ} 50^{\prime} 0,94^{\prime \prime} \mathrm{O}\end{array}$ & & 52 & 474,03 & 108,7 & 39 \\
\hline Sapiranga & SA & $\begin{array}{l}29^{\circ} 38^{\prime} 3,13^{\prime \prime} \mathrm{S} \\
51^{\circ} 0{ }^{\prime} 23,58^{\prime \prime} \mathrm{O}\end{array}$ & $\begin{array}{l}\text { Rural - } \\
\text { Urbano }\end{array}$ & 32 & 542,14 & 138 & 43 \\
\hline Estância Velha & EV & $\begin{array}{c}29^{\circ} 39^{\prime} 5,28^{\prime \prime} \mathrm{S} \\
51^{\circ} 10^{\prime} 21,89^{\prime \prime} \mathrm{O}\end{array}$ & & 45 & 816,42 & 52,1 & 45 \\
\hline Novo Hamburgo & $\mathrm{NH}$ & $\begin{array}{c}29^{\circ} 40^{\prime} 57,08^{\prime \prime} \mathrm{S} \\
51^{\circ} 7{ }^{\prime} 41,82^{\prime \prime} \mathrm{O}\end{array}$ & & 29 & $1,067,55$ & 223,8 & 85 \\
\hline São Leopoldo & SL & $\begin{array}{c}29^{\circ} 45^{\prime} 52,12^{\prime \prime} \mathrm{S} \\
51^{\circ} 9^{\prime} 0,05^{\prime \prime} \mathrm{O}\end{array}$ & $\begin{array}{l}\text { Urbano - } \\
\text { Industrial }\end{array}$ & 15 & $2,083,82$ & 102,7 & 61 \\
\hline Canoas & $\mathrm{CN}$ & $\begin{array}{l}29^{\circ} 54^{\prime} 55,38^{\prime \prime} \mathrm{S} \\
51^{\circ} 10^{\prime} 13,11^{\prime \prime} \mathrm{O}\end{array}$ & & 15 & $2,470,15$ & 131 & 83 \\
\hline
\end{tabular}

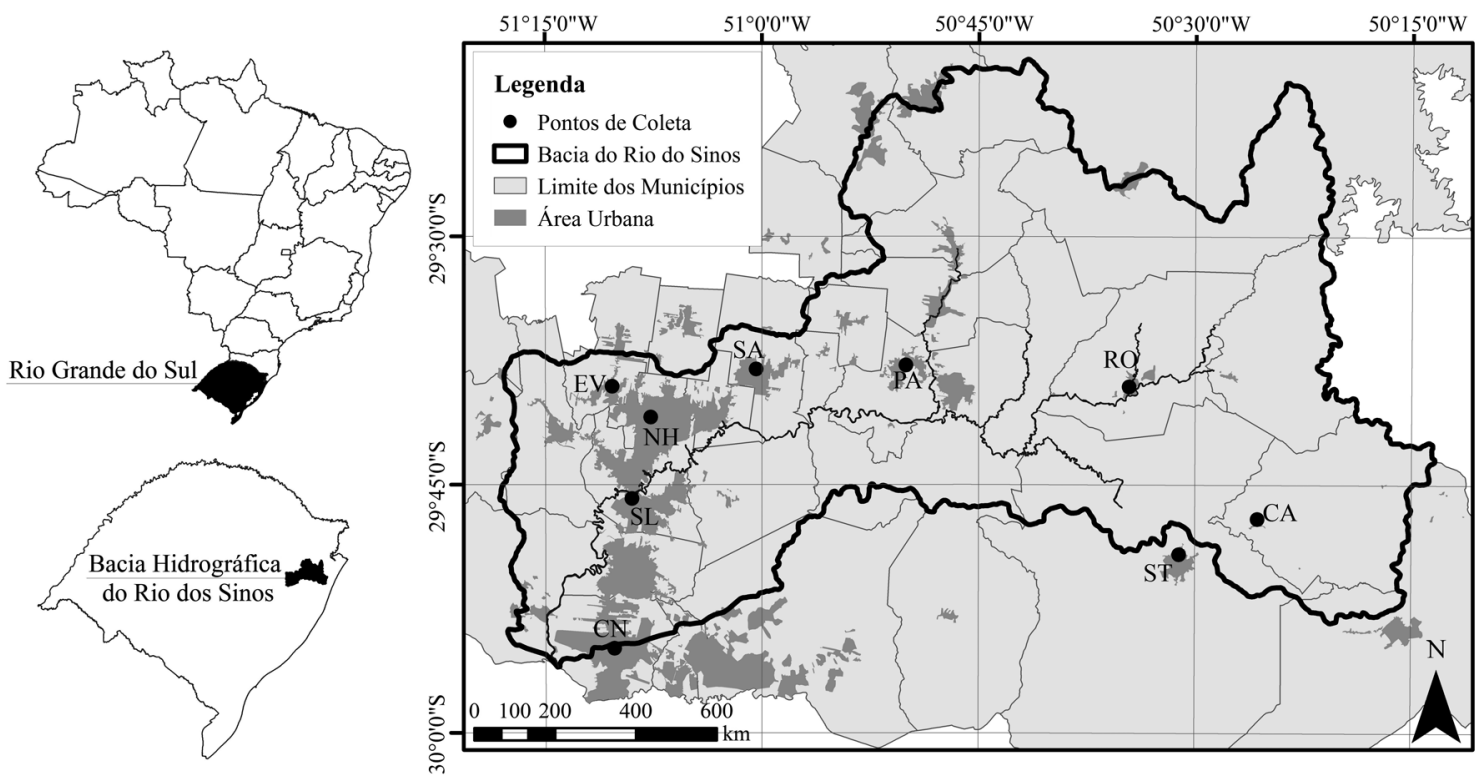

Figura 1 - Localização dos municípios estudados na BHRS, RS, Brasil. CA = Caraá; ST = Santo Antônio da Patrulha; RO $=$ Rolante; $\mathrm{PA}=$ Parobé; $\mathrm{SA}=$ Sapiranga $\mathrm{EV}=$ Estância Velha; $\mathrm{NH}=$ Novo Hamburgo; $\mathrm{SL}=$ São Leopoldo; $\mathrm{CN}=\mathrm{Canoas}$. Figure 1 - Location of the studied municipalities in the BHRS, RS, Brazil. CA = Caraá; ST = Santo Antônio da Patrulha; RO = Rolante; PA $=$ Parobé; $\mathrm{SA}=$ Sapiranga; EV = Estância Velha; NH = Novo Hamburgo; SL = São Leopoldo; CN = Canoas. 


\section{Amostragem}

Para a amostragem da comunidade de liquens, em cada município, foram analisadas 10 árvores isoladas (forófitos), apresentando circunferência a altura do peito (CAP) $>20 \mathrm{~cm}$, fuste reto e sem ramificações até $1,50 \mathrm{~m}$ de altura. Os forófitos estavam localizados em praças ou parques. Ao total, foram amostradas 90 árvores (Tab. 2).

Em cada forófito, a amostragem dos liquens foi feita entre 50 e $150 \mathrm{~cm}$ acima do solo, em intervalos de $10 \mathrm{~cm}$, conforme o método do elástico (Marcelli 1992), que consiste em posicionar um elástico ao redor do tronco, subdividido igualmente em 20 divisões que perfazem $100 \%$ de cobertura. O comprimento do elástico varia conforme a circunferência do tronco.

A cobertura de uma espécie de liquen foi estimada com base no número de divisões ocupadas por ela ao longo do elástico. A cobertura final da espécie por forófito considerou a soma do percentual de pontos tocados por ela nos 11 níveis de altura. A cobertura total por município considerou a soma da cobertura da espécie nos 10 forófitos amostrados.

Os espécimes de liquens foram identificados no local e/ou coletados para posterior identificação

Tabela 2 - Forófitos amostrados nos municípios estudados na BHRS, RS, Brasil.

Table 2 - Sampled phorophytes in the studied municipalities in the BHRS, RS, Brazil.

\begin{tabular}{|c|c|c|c|c|c|c|c|c|c|c|}
\hline Família & Espécie & $\mathbf{C A}$ & ST & RO & PA & SA & EV & NH & SL & $\mathbf{C N}$ \\
\hline Anacardiaceae & Schinus terebinthifolius Raddi & & & & 1 & & & 1 & & \\
\hline Bignoniaceae & Handroanthus chrysotrichus (Mart. ex DC.) Mattos & & & 1 & & & 1 & & 1 & 4 \\
\hline Bignoniaceae & Handroanthus heptaphyllus (Vell.) Mattos & & & 7 & 5 & 2 & 3 & 4 & 1 & 4 \\
\hline Bignoniaceae & Handroanthus umbellatus (Sond.) Mattos & & & & 1 & & & & & \\
\hline Bignoniaceae & Handroanthus sp. & & & 1 & & & & & & \\
\hline Bignoniaceae & Jacaranda mimosifolia D. Don. & & & & & & 2 & & & \\
\hline Euphorbiaceae & Aleurites moluccanus (L.) Willd. & & & & & & 1 & 1 & & \\
\hline Euphorbiaceae & Sapium glandulosum (L.) Morong. & & & & 1 & & & & & \\
\hline Fabaceae & Caesalpinia pluviosa DC. & & & & & 4 & & & & \\
\hline Fabaceae & Hymenaea sp & & & 1 & & & & & & \\
\hline Fabaceae & $\begin{array}{l}\text { Muellera campestris (Mart. ex Benth.) } \\
\text { M.J. Silva \& A.M.G. Azevedo }\end{array}$ & 1 & & & & & & & & \\
\hline Fabaceae & Peltophorum dubium (Spreng.) Taub. & & & & 2 & 2 & & & 7 & \\
\hline Fabaceae & $\begin{array}{l}\text { Senna macranthera (DC. ex Collad.) } \\
\text { H.S. Irwin \& Barneby }\end{array}$ & & & & & & 1 & & & \\
\hline Lauraceae & Cinnamomum verum J.S. Presl & & & & & & & 1 & & \\
\hline Malvaceae & Brachychiton populneus (Schott et Endl.) R.Br. & & & & & 2 & & & & \\
\hline Malvaceae & Luehea divaricata Mart. \& Zucc. & & & & & & 1 & & & \\
\hline Oleaceae & Ligustrum lucidum Aiton & & 3 & & & & & & & 2 \\
\hline Primulaceae & Myrsine umbellata Mart. & & 6 & & & & & & & \\
\hline Rhamnaceae & Hovenia dulcis Thunb. & & & & & & 1 & & & \\
\hline Rosaceae & Eriobotrya japonica Lindl. & & & & & & & 3 & & \\
\hline Salicaceae & Casearia sylvestris Sw. & & 1 & & & & & & & \\
\hline Sapindaceae & Cupania vernalis Cambess. & 3 & & & & & & & & \\
\hline \multirow[t]{4}{*}{ Verbenaceae } & Verbenoxylum reitzii (Mold.) Tronc. & 4 & & & & & & & & \\
\hline & N.I.1 & 1 & & & & & & & & \\
\hline & N.I.2 & 1 & & & & & & & & \\
\hline & N.I.3 & & & & & & & & 1 & \\
\hline
\end{tabular}


em laboratório. Para tanto, foram utilizadas técnicas convencionais com microscópio estereoscópio e ótico, por meio de análises morfológicas e químicas, incluindo testes de coloração no córtex e na medula. Também foi utilizado o teste de fluorescência por exposição à luz ultravioleta (Teste UV), para observação das variações das fluorescências do córtex e da medula (Huneck \& Yoshimura 1996). Para identificação taxonômica também foi utilizada bibliografia especializada (Brodo et al. 2001; Cáceres 2007; Fleig et al. 2008; Aptroot et al. 2009; Lücking et al. 2009; Spielmann \& Marcelli 2009), auxílio de especialistas e consulta a material herborizado. O material coletado foi herborizado e incluído na coleção do Herbário Prof. Dr. Alarich Rudolf Holger Schultz (HAS), do Museu de Ciências Naturais da Fundação Zoobotânica do Rio Grande do Sul, HAS No 92758 a 92989.

Análise de dados

Para cada município foram analisados os parâmetros de riqueza, de frequência absoluta (FA) e relativa (FR) e de cobertura absoluta (CA) e relativa (CR). $\mathrm{O}$ índice de valor de importância (IVI) das espécies foi calculado somando-se os valores de CR e FR de cada espécie $(\Sigma=200)$.

Riqueza e cobertura das espécies por forófitos de cada município foram submetidos ao teste de normalidade de Shapiro-Wilk, e comparados pela análise de variância (ANOVA), seguida do teste de Tukey a 5\% de probabilidade. Estas análises foram realizadas no programa estatístico Paleontological Statistics Software Package for Education and Data Analysis (PAST), versão 3.01 (Hammer et al. 2001).

Para definir o gradiente da composição da comunidade de liquens, foi realizada uma análise de NMDS (escalonamento multidimensional não métrico), baseada em uma matriz da cobertura de espécies por unidade amostral. Os dados foram submetidos a 500 interações usando a medida de distância de Bray-Curtis, sendo que o menor estresse foi escolhido. O teste de Monte Carlo foi realizado para testar a significância da ordenação quando comparado com dados aleatórios, utilizando o software PC-ORD 6.0 (Mccune \& Mefford 2011).

\section{Resultados}

Ao total foram inventariadas 215 espécies, pertencentes a 52 gêneros e 27 famílias (Tab. 3, disponível no link <https://figshare.com/ s/7552d3a29e032053914a> - DOI: 10.6084/ m9.figshare.6121451). Nove espécies registradas neste estudo são novas ocorrências, sendo quatro delas para o Brasil: Lecanora elapheia Stizenb., Lithothelium illotum (Vain.) Aptroot, Pyrenula acutispora Kalb \& Hafellner e Strigula muriconidiata Aptroot, L. I. Ferraro \& M. Cáceres; e cinco para o Rio Grande do Sul: Chrysothrix xanthina (Vain.) Kalb, Coccocarpia prostrata Lücking, Aptroot \& Sipman, Lecanora tropica Zahlbr., Anisomeridium subnectendum (Nyl.) R.C. Harris e Lepraria sipmaniana (Kümmerl. \& Leuckert) Kukwa.

Dentre as famílias representadas, Parmeliaceae foi a mais rica com 55 espécies, seguida por Physciaceae e por Graphidaceae, ambas com 34. Apenas 22 espécies ocorreram em cinco ou mais municípios, sendo que Crespoa carneopruinata (Zahlbr.) Lendemer \& B.P. Hodk. foi comum nos nove, seguida por Canoparmelia texana (Tuck.) Elix \& Hale e Heterodermia albicans (Pers.) Swinscow \& Krog que estiveram presentes em oito (Tab. 3, disponível no link < https://figshare. com/s/7552d3a29e032053914a $>$ - DOI: 10.6084/ m9.figshare.6121451). A espécie com maior frequência foi $C$. carneopruinata encontrada em 43 forófitos. Os municípios localizados na matriz rural apresentaram um maior número de espécies exclusivas, 76 ao total, ao contrário dos localizados na matriz urbano/industrial que apresentaram 21 espécies (Tab. 4).

Quanto aos grupos morfológicos, 52\% das espécies estão representadas por liquens foliosos, $45 \%$ crostosos, $2 \%$ fruticosos e apenas $1 \%$ esquamulosos (Tab. 4). Somente 11 espécies pertencentes aos gêneros Coccocarpia (2) e Leptogium (9) apresentaram associação com cianobactérias (5\%), sendo que as demais (95\%) estão associadas a clorofíceas.

A maior riqueza total de espécies de liquens foi encontrada no município de Sapiranga (rural/ urbano) (76), seguida por Caraá (rural) (63), enquanto que a menor foi registrada em Canoas (urbano/industrial) (27) (Tab. 4). A maior riqueza média de espécies por forófito também foi obtida em Sapiranga, sendo estatisticamente igual à de Santo Antônio da Patrulha, Parobé, Caraá e Rolante, municípios localizados nas matrizes rural e rural/urbano. Por outro lado, a menor média de riqueza por forófito foi registrada $\mathrm{em}$ Canoas (urbano/industrial), sendo estatisticamente diferente de Sapiranga (Fig. 2a).

Em relação ao percentual de cobertura de liquens, o maior valor foi encontrado em Caraá, 
Tabela 4 - Riqueza, cobertura e espécies exclusivas encontradas nos municípios estudados na BHRS, RS, Brasil. Table 4 - Species richness, coverage and exclusive species found in the studied municipalities in the BHRS, RS, Brazil.

\begin{tabular}{lcccc}
\hline Município & Matriz & Riqueza & Cobertura (\%) & $\begin{array}{c}\text { No espécies } \\
\text { exclusivas }\end{array}$ \\
\hline Caraá & & 63 & 47 & 38 \\
Santo Antônio da Patrulha & Rural & 61 & 37 & 22 \\
Rolante & & 58 & 20 & 16 \\
\hline Parobé & Rural / & 47 & 35 & 3 \\
Sapiranga & Urbano & 76 & 37 & 23 \\
Estância Velha & & 35 & 13 & 8 \\
\hline Novo Hamburgo & & 32 & 15 & 8 \\
São Leopoldo & Urbano / Industrial & 35 & 23 & 8 \\
Canoas & & 27 & 37 & 5 \\
\hline
\end{tabular}

(47\%) e o menor em Estância Velha (13\%), respectivamente, em matriz rural e rural/urbano (Tab. 4). Quanto à cobertura de liquens por forófito, a maior média foi registrada em Caraá (rural), sendo significativamente diferente de Rolante, Estância Velha e Novo Hamburgo, localizados nas matrizes rural/urbano e urbano/industrial (Fig. 2b).

Em relação à estrutura da comunidade, Canoparmelia texana e Crespoa carneopruinata estiveram entre as cinco espécies com maior IVI, em cinco dos nove municípios. Heterodermia albicans ocorreu em quatro municípios amostrados (Tab. 5). Canoparmelia texana ficou em primeiro lugar no IVI em quatro dos cinco municípios em que ocorreu. Physciaceae e Parmeliaceae foram às famílias mais ricas, considerando as espécies com maior IVI nos municípios e apresentaram 11 e sete espécies, respectivamente.

A variação na composição da comunidade de liquens foi explicada por três eixos da NMDS com um estresse mínimo final de $21,7 \%(\mathrm{p}=0,006)$. O primeiro eixo explicou $18,9 \%$ da variação na
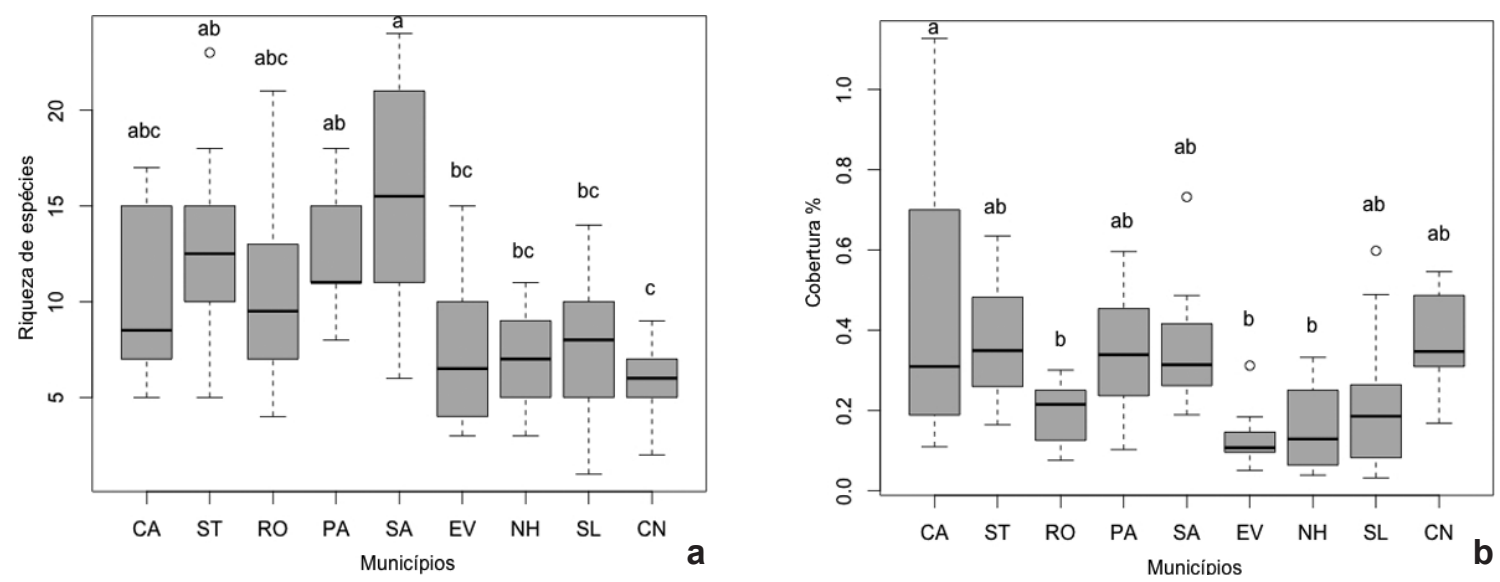

Figura 2 - a,b. Boxplot de liquens nos municípios estudados na BHRS, RS, Brasil - a. da riqueza; b. percentual de cobertura. Linhas dentro dos boxes representam a mediana e as barras o erro padrão. Letras diferentes representam diferenças significativas $(\mathrm{P}<0,05)$. CA = Caraá; $\mathrm{ST}=$ Santo Antônio da Patrulha; $\mathrm{RO}=$ Rolante; $\mathrm{PA}=$ Parobé; $\mathrm{SA}=$ Sapiranga; EV = Estância Velha; $\mathrm{NH}=$ Novo Hamburgo; $\mathrm{SL}=$ São Leopoldo; $\mathrm{CN}=$ Canoas.

Figure 2 -a,b. Boxplot of lichen species in the studied municipalities, BHRS, RS, Brazil - a. richness; b. coverage percentage. The lines inside the boxes represent the median and the bars, the standard error. Different letters indicate significant differences $(\mathrm{P}<0.05)$. CA $=$ Caraá; $\mathrm{ST}=\mathrm{Santo}$ Antônio da Patrulha; $\mathrm{RO}=$ Rolante; $\mathrm{PA}=$ Parobé; $\mathrm{SA}=$ Sapiranga; $\mathrm{EV}=$ Estância Velha; $\mathrm{NH}=$ Novo Hamburgo; $\mathrm{SL}=\mathrm{São} \mathrm{Leopoldo;} \mathrm{CN}=\mathrm{Canoas}$. 
Tabela 5 - Relação das cinco espécies com maior IVI nos municípios estudados na BHRS, RS, Brasil. NF = número de forófitos ocorrentes; $\mathrm{FA}=$ frequência absoluta $(\%) ; \mathrm{FR}=$ frequência relativa (\%); $\mathrm{CA}=$ cobertura absoluta; $\mathrm{CR}$ $=$ cobertura relativa $(\%) ;$ IVI $=$ índice de valor de importância $(\%)$.

Table 5 - Relation of the five species with highest IVI in the studied municipalities from the BHRS, RS, Brazil. NF = number of occurring phorophytes; $\mathrm{FA}=$ absolute frequency $(\%) ; \mathrm{FR}=$ relative frequency $(\%) ; \mathrm{CA}=$ absolute coverage; $\mathrm{CR}=$ relative coverage $(\%) ;$ IVI $=$ index of importance value $(\%)$.

\begin{tabular}{|c|c|c|c|c|c|c|c|}
\hline Município & Espécies & NF & FA & FR & CA & $\mathbf{C R}$ & IVI \\
\hline \multirow[t]{6}{*}{ Caraá } & Herpothallon echinatum & 5 & 50 & 4,81 & 13,80 & 29,40 & 34,21 \\
\hline & Heterodermia obscurata & 7 & 70 & 6,73 & 4,09 & 8,72 & 15,45 \\
\hline & Coenogonium cf. bacilliferum & 7 & 70 & 6,73 & 1,50 & 3,20 & 9,93 \\
\hline & Leptogium aff. cyanescens & 4 & 40 & 3,85 & 2,04 & 4,35 & 8,20 \\
\hline & cf, Cryptothecia sp. & 4 & 40 & 3,85 & 1,95 & 4,17 & 8,01 \\
\hline & & & & \multicolumn{2}{|c|}{ Somatório $(\Sigma)$} & 49,83 & 75,79 \\
\hline \multirow{6}{*}{$\begin{array}{l}\text { Santo Antônio da } \\
\text { Patrulha }\end{array}$} & Heterodermia albicans & 6 & 60 & 4,76 & 3,82 & 10,33 & 15,10 \\
\hline & Physcia tribacoides & 5 & 50 & 3,97 & 2,63 & 7,11 & 11,08 \\
\hline & Physcia aipolia & 7 & 70 & 5,56 & 1,77 & 4,80 & 10,35 \\
\hline & Heterodermia sp.1 & 3 & 30 & 2,38 & 2,80 & 7,57 & 9,95 \\
\hline & Canoparmelia caroliniana & 6 & 60 & 4,76 & 1,27 & 3,44 & 8,21 \\
\hline & & & & \multicolumn{2}{|c|}{ Somatório $(\Sigma)$} & 33,26 & 54,68 \\
\hline \multirow[t]{6}{*}{ Rolante } & Parmotrema reticulatum & 8 & 80 & 7,62 & 3,27 & 16,40 & 24,02 \\
\hline & Heterodermia albicans & 7 & 70 & 6,67 & 2,55 & 12,76 & 19,42 \\
\hline & Crespoa carneopruinata & 5 & 50 & 4,76 & 2,32 & 11,62 & 16,38 \\
\hline & Heterodermia obscurata & 6 & 60 & 5,71 & 1,41 & 7,06 & 12,78 \\
\hline & Parmotrema tinctorum & 6 & 60 & 5,71 & 0,52 & 2,62 & 8,33 \\
\hline & & & & \multicolumn{2}{|c|}{ Somatório $(\Sigma)$} & 50,46 & 80,93 \\
\hline \multirow[t]{6}{*}{ Parobé } & Canoparmelia texana & 6 & 60 & 4,76 & 6,11 & 17,45 & 22,21 \\
\hline & Crespoa carneopruinata & 9 & 90 & 7,14 & 3,32 & 9,47 & 16,61 \\
\hline & Physcia krogiae & 8 & 80 & 6,35 & 3,57 & 10,18 & 16,53 \\
\hline & Dirinaria applanata & 6 & 60 & 4,76 & 3,04 & 8,67 & 13,44 \\
\hline & Pyxine albovirens & 6 & 60 & 4,76 & 2,09 & 5,97 & 10,73 \\
\hline & & & & \multicolumn{2}{|c|}{ Somatório $(\Sigma)$} & 51,74 & 79,52 \\
\hline \multirow[t]{6}{*}{ Sapiranga } & Canoparmelia texana & 6 & 60 & 3,87 & 8,48 & 23,16 & 27,04 \\
\hline & Dirinaria applanata & 7 & 70 & 4,52 & 3,16 & 8,63 & 13,15 \\
\hline & Parmotrema austrosinense & 7 & 70 & 4,52 & 2,66 & 7,27 & 11,78 \\
\hline & Parmotrema lindmanii & 5 & 50 & 3,23 & 2,41 & 6,58 & 9,81 \\
\hline & Parmotrema tinctorum & 4 & 40 & 2,58 & 1,59 & 4,35 & 6,93 \\
\hline & & & & \multicolumn{2}{|c|}{ Somatório $(\Sigma)$} & 49,99 & 68,70 \\
\hline \multirow[t]{6}{*}{ Estância Velha } & Physcia krogiae & 7 & 70 & 9,33 & 2,64 & 19,83 & 29,16 \\
\hline & Physcia poncinsii & 2 & 20 & 2,67 & 2,23 & 16,75 & 19,42 \\
\hline & Canoparmelia texana & 4 & 40 & 5,33 & 1,50 & 11,28 & 16,62 \\
\hline & Heterodermia albicans & 6 & 60 & 8,00 & 0,73 & 5,47 & 13,47 \\
\hline & Crespoa carneopruinata & 4 & 40 & 5,33 & 0,66 & 4,96 & 10,29 \\
\hline & & & & \multicolumn{2}{|c|}{ Somatório $(\Sigma)$} & 58,29 & 88,96 \\
\hline
\end{tabular}




\begin{tabular}{llcccccc}
\hline Município & Espécies & NF & FA & FR & CA & CR & IVI \\
\hline Novo Hamburgo & Physcia krogiae & 9 & 90 & 12,68 & 1,91 & 12,56 & 25,23 \\
& Physcia poncinsii & 5 & 50 & 7,04 & 2,43 & 15,99 & 23,04 \\
& Crespoa carneopruinata & 5 & 50 & 7,04 & 1,41 & 9,27 & 16,31 \\
& Physcia atrostriata & 6 & 60 & 8,45 & 0,86 & 5,68 & 14,13 \\
& Punctelia sp.7 & 1 & 10 & 1,41 & 1,68 & 11,06 & 12,47 \\
& & & & Somatório $(\Sigma)$ & 54,56 & 91,18 \\
\hline São Leopoldo & Canoparmelia texana & 7 & 70 & 9,09 & 7,20 & 31,89 & 40,98 \\
& Dirinaria picta & 7 & 70 & 9,09 & 2,75 & 12,17 & 21,26 \\
& Crespoa carneopruinata & 6 & 60 & 7,79 & 1,73 & 7,65 & 15,44 \\
& Graphis furcata & 3 & 30 & 3,90 & 1,41 & 6,24 & 10,13 \\
& Heterodermia albicans & 4 & 40 & 5,19 & 1,11 & 4,93 & 10,12 \\
& & & & & Somatório $(\Sigma)$ & 62,88 & 97,94 \\
\hline Canoas & Canoparmelia texana & 8 & 80 & 14,29 & 20,57 & 55,90 & 70,19 \\
& Parmotrema tinctorum & 6 & 60 & 10,71 & 5,43 & 14,76 & 25,48 \\
& Parmotrema clavuliferum & 6 & 60 & 10,71 & 2,20 & 5,99 & 16,71 \\
& Dirinaria picta & 5 & 50 & 8,93 & 0,65 & 1,78 & 10,71 \\
& Punctelia sp.4 & 1 & 10 & 1,79 & 2,91 & 7,91 & 9,69 \\
& & & & & Somatório $(\Sigma)$ & 86,35 & 132,78 \\
\hline
\end{tabular}

comunidade de liquens, o segundo $18,3 \%$ e o terceiro apenas $12,4 \%$ (Fig. 3). Os municípios inseridos na matriz rural ficaram, na maioria, à direita da ordenação, e os localizados na matriz urbano/industrial à esquerda, evidenciando assim o gradiente representado pelas matrizes.

\section{Discussão}

Foi observado um decréscimo da riqueza de espécies da matriz rural em direção à urbano/ industrial, com exceção de Sapiranga (rural/ urbano), que foi o município com maior riqueza. Becker et al. $(2015,2017)$ e Rocha-Uriartt et al. (2015) ao estudarem epífitos vasculares na zona urbana e em mata ciliar em municípios da BHRS também encontraram este mesmo padrão de decréscimo da riqueza de espécies.

Alguns aspectos da urbanização promovem a perda de diversidade de espécies como a redução e fragmentação da área disponível e as alterações nos fatores abióticos como a precipitação, a umidade, a velocidade e a direção dos ventos (Foley et al. 2005; McKinney 2008; Pinho et al. 2017). Lättman et al. (2014) verificaram também uma redução na riqueza de espécies de liquens em áreas urbanas em relação às rurais. No entanto, os efeitos de níveis moderados de urbanização, como o apresentado em Sapiranga, município com maior riqueza de espécies, variam significativamente entre os grupos. Estudos com plantas demostraram uma tendência menos consistente de reduzir a riqueza de espécies, com urbanização moderada (McKinney 2008).

Foi possível observar que municípios com matriz rural (ST), rural/urbano (SA) ou urbano/industrial $(\mathrm{CN})$ apresentaram similaridade quanto ao valor de cobertura das espécies de liquens. Segundo Lättman et al. (2014) a riqueza e a cobertura geralmente decrescem com o aumento da urbanização, exceto pela presença de espécies invasoras ou generalistas, uma vez que essas podem apresentar maior adaptação e tolerância a zonas urbanas. Canoparmelia texana é uma espécie generalista que esteve em primeiro lugar entre aquelas com maior IVI, em quatro municípios. Esta espécie apresentou alto valor de cobertura, correspondendo a $56 \%$ em $\mathrm{CN}$ e $32 \%$ em SL. Ela é uma das espécies mais amplamente distribuídas pelo território brasileiro, sendo facilmente encontrada em áreas urbanas (Marcelli 1998; Fuga et al. 2008). Característica de ambientes alterados, Canoparmelia texana pode ocupar o espaço preenchido anteriormente por outras espécies menos resistentes às mudanças ambientais (Martins et al. 2008; Kaffer et al. 2011). 
Ela também apresentou maior IVI no estudo de Käffer et al. (2011) realizado em área urbana de Porto Alegre, próxima à matriz urbano/industrial do presente estudo.

Os municípios localizados na matriz urbano/ industrial (Novo Hamburgo, São Leopoldo e Canoas), além de apresentarem menor riqueza demostraram uma simplificação na estrutura da comunidade. As cinco espécies mais importantes na comunidade apresentam alto IVI e de cobertura relativa, evidenciando que espécies tolerantes e generalistas são mais comuns em ambientes mais urbanizados.
Foi possível verificar mudanças em relação à composição das espécies nas comunidades de liquens com o aumento do percentual de área urbana no entorno dos municípios estudados. As áreas localizadas na matriz rural apresentaram um maior número de espécies exclusivas, incluindo representantes de Crocodia, Herpothallon e Ricasolia entre outros. Espécies destes gêneros são comuns em florestas tropicais preservadas, características de ambientes sombreados e comumente encontradas crescendo em árvores com cascas úmidas (Aptroot et al. 2009; Käffer et al. 2010; Kaffer et al. 2015). A presença destas

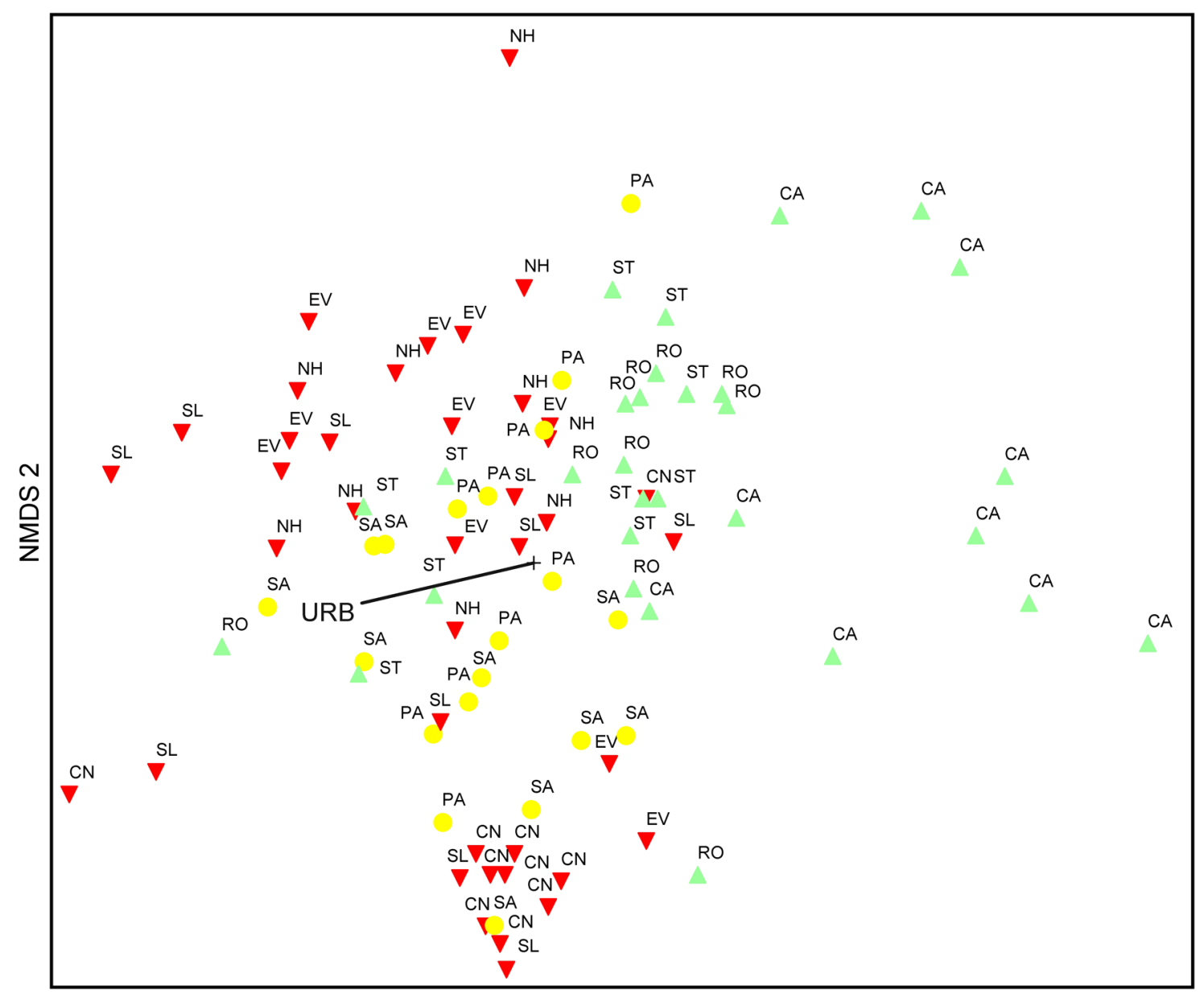

NMDS 1

Figura 3 - Ordenação multidimensional não métrica (NMDS) de acordo com a composição de espécies de liquens por matriz da BHRS. $\Delta=$ matriz rural, $\odot=$ matriz rural/urbano, $\boldsymbol{\nabla}=$ matriz urbano/industrial, $\mathrm{CA}=\mathrm{Caraá}, \mathrm{ST}=\mathrm{Santo}$ Antônio da Patrulha, RO = Rolante, $\mathrm{PA}=$ Parobé, $\mathrm{SA}=$ Sapiranga, EV = Estância Velha, NH = Novo Hamburgo, $\mathrm{SL}=$ São Leopoldo e $\mathrm{CN}=$ Canoas, URB $=$ urbanização.

Figure 3 - Non-metric multidimensional scaling (NMDS) according to the lichen species composition by matrix of the BHRS. $\triangle=$ rural matrix, = rural/urban matrix, $\boldsymbol{\nabla}=$ urban/industrial matrix, $\mathrm{CA}=$ Caraá, $\mathrm{ST}=$ Santo Antônio da Patrulha, $\mathrm{RO}=\mathrm{Rolante}, \mathrm{PA}$ $=$ Parobé, $\mathrm{SA}=$ Sapiranga, EV = Estância Velha, $\mathrm{NH}=$ Novo Hamburgo, $\mathrm{SL}=$ São Leopoldo e $\mathrm{CN}=$ Canoas, URB = urbanization. 
espécies em municípios da matriz rural pode estar associada às condições mais favoráveis para seu estabelecimento, tal como maior precipitação e por apresentar áreas mais conservadas em seu entorno, fatores já demostrados nos estudos realizados em municípios localizados nesta matriz por Cassanego et al. (2015), Rocha-Uriartt et al. (2015) e Becker et al. (2017).

Mudanças na estrutura da paisagem são conhecidas por afetar a qualidade dos hábitats e podem ser um fator importante para explicar a diferenciação no padrão de diversidade e composição de algumas espécies de liquens (Belinchón et al. 2009; Ardelean et al. 2015). A composição das espécies que refletem a biodiversidade de uma cidade não é a mesma encontrada em áreas rurais ou naturais (Pinho et al. 2017). A urbanização tem uma forte influência na formação de comunidades de liquens (Pinho et al. 2016), sendo que a expansão urbana resulta em menor densidade de espécies em comparação com áreas rurais (Seto et al. 2016; Aronson et al. 2014), tal como verificado neste estudo.

\section{Conclusões}

Os parâmetros avaliados indicaram que os municípios inseridos em uma matriz rural apresentam melhores condições para o desenvolvimento da comunidade de liquens. Foi possível verificar uma simplificação na estrutura das comunidades nos municípios inseridos em matriz urbano/industrial, pois poucas espécies tiveram alta dominância e juntas representaram um alto valor de importância.

Em relação à composição das espécies, foi observada maior homogeneidade entre áreas mais próximas, localizadas na matriz urbano/industrial, sendo estas diferentes das áreas da matriz rural. Desta forma ficou demonstrado que o aumento da urbanização é um importante fator para a diferenciação das comunidades de liquens em municípios localizados na BHRS.

O grande número de espécies de liquens inventariado, incluindo novas ocorrências para o Brasil e para o Rio Grande do Sul, demonstra a importância da manutenção e da implementação de áreas verdes urbanas, com vegetação nativa, que proporciona diferentes habitats para a manutenção de toda a biota.

\section{Agradecimentos}

Os autores agradecem à Coordenação de Aperfeiçoamento de Pessoal de Nível Superior
(CAPES), a concessão da bolsa de Mestrado ao primeiro autor; à Universidade Feevale e Fundação Zoobotânica do RS, a infraestrutura; aos bolsistas do Laboratório de Botânica e de Liquenologia, o auxílio em campo; aos liquenólogos, André Aptroot, Emerson Gumbosky, Márcia Käffer, Marcos Kitaura, Patrícia Jungbluth e Shirley Feuerstein, o auxílio na identificação e confirmação de algumas espécies.

\section{Referências}

Anschau C (2016) Atlas do Projeto Verdesinos. Editora Anschau, Porto Alegre. Pp. 116.

Aptroot A, Thor G, Lücking R, Elix JA \& Chaves JL (2009) The lichen genus Herpothallon reinstated. Bibliotheca Lichenologica 99: 19-66.

Ardelean IO, Keller C \& Scheidegger C (2015) Effects of management on lichen species richness, ecological traits and community structure in the Rodnei Mountains National Park (Romania). PLoS ONE 10: 1-16.

Aronson MFJ, La Sorte FA \& Nilon CH et al. (2014) A global analysis of the impacts of urbanization on bird and plant diversity reveals key anthropogenic drivers. Proceedings of the Royal Society B 281: 20133330.

Asta J, Erhardt W, Ferretti M, Fornasier F, Kirschbaum U, Nimis PL, Purvis OW, Pirintsos S, Scheidegger C, Van Haluwyn C \& Wirth V (2002) European guideline for mapping lichen diversity as an indicator of environmental stress. British Lichen Society, London. Pp. 20.

Becker DFP, Linden R \& Schmitt JL (2017) Richness, coverage and concentration of heavy metals in vascular epiphytes along an urbanization gradiente. Science of the Total Environment 584-585: 48-54.

Becker DFP, Padoin TOH, Nascimento CA, Robalski JL, Linden R \& Schmitt JL (2015) Riqueza e composição de epífitos vasculares em áreas urbanas da Bacia Hidrográfica do Rio dos Sinos, RS, Brasil. Pesquisas, Botânica 68: 227-238.

Belinchón R, Martínez I, Otálora MAG, Aragón G, Dimas J \& Escudero A (2009) Fragment quality and matrix affect epiphytic performance in a mediterranean forest landscape. American Journal of Botany 96: 1974-1982.

Brodo IM, Sharnoff SD \& Sharnoff S (2001) Lichens of North America. Yale University Press, New Haven. 795p.

Cáceres MES (2007) Corticolous crustose and microfoliose lichens of northeastern Brazil. Libri Botanici 22: 1-168.

Cáceres MES, Lücking R \& Rambold G (2008) Corticolous microlichens in north-eastern Brazil: habitat differentiation between coastal Mata Atlântica, Caatinga and Brejos de Altitude. The Bryologist 111: 98-117. 
Cassanego MBB, Sasamori MH, Petry CT \& Droste A (2015) Biomonitoring the genotoxic potential of the air on Tradescantia pallida var. purpurea under climatic conditions in the Sinos River basin, Rio Grande do Sul, Brazil. Brazilian Journal of Biology 75: 79-87.

Conti ME \& Cecchetti G (2001) Biological monitoring: lichens as bioindicators of air pollution assessment - a review. Environmental Pollution 114: 471-492.

FEPAM - Fundação Estadual de Proteção Ambiental Henrique Luiz Roessler - RS (2016) Qualidade ambiental - região hidrográfica do Guaíba. Disponível em <http://www.fepam.rs.gov.br/ qualidade/qualidade sinos/sinos.asp $>$. Acesso em 20 junho 2016.

Figueiredo JAS, Drumm E, Rodrigues MAS \& Spilki FR (2010) The Rio dos Sinos watershed: an economic and social space and its interface with environmental status. Brazilian Journal of Biology 70: 1131-1136.

Fleig M, Grüninger W, Mayer W-E \& Hampp R (2008) Liquens da floresta com Araucária no Rio Grande do Sul. Pró-mata: guia de campo n ${ }^{\circ} 3$. University of Tübingen. ediPUCRS, Porto Alegre. Pp. 219.

Foley JA, DeFries R, Asner GP, Barford C, Bonan G, Carpenter SR, Chapin FS, Coe MT, Daily GC, Gibbs HK, Helkowski JH, Holloway T, Howard EA, Kucharik CJ, Monfreda C, Patz JA, Prentice C, Ramankutty N \& Snyder P (2005) Global consequences of land use. Science 309: 570-574

Fuga A, Saiki M, Marcelli MP \& Saldiva PHN (2008) Atmospheric pollutants monitoring by analysis of epiphytic lichens. Environmental Pollution 151: 334-340.

Gagne SA \& Fahrig L (2011) Do birds and beetles show similar responses to urbanization? Ecological Applications 21: 2297-2312.

Hammer O, Harper DAT \& Ryan PD (2001) PAST: paleontological statistics software package for education and data analysis. Palaeontologia Electronica 4: 9.

Huneck S \& Yoshimura I (1996) Identification of lichen substances. Springer-Verlag, Berlin, Heidelberg. 493p.

Käffer MI, Marcelli MP \& Ganade G (2010) Distribution and composition of the lichenized mycota in a landscape mosaic of southern Brazil. Acta Botanica Brasilica 24: 790-802.

Käffer MI, Alves C, Cáceres MES, Martins SMA \& Vargas VMF (2011) Caracterização da comunidade liquênica corticícola de Porto Alegre e áreas adjacentes, RS, Brasil. Acta Botanica Brasilica 25: 832-844.

Käffer MI, Lemos AT, Apel MA, Rocha JV, Martins SMA \& Vargas VMF (2012) Use of bioindicators to evaluate air quality and genotoxic compounds in an urban environment in Southern Brazil. Environmental Pollution 163: 24-31.
Käffer MI, Martins SMA, Dantas RV \& Maciel FC (2015) Composição da comunidade liquênica em floresta ribeirinha na APA do Ibirapuitã, RS, Brasil. Hoehnea 42: 273-288.

Koch NM, MalufRW \& Martins SMA(2012) Comunidade de liquens foliosos em Piptocarpha angustifolia Dusén ex Malme (Asteraceae) em área de Floresta Ombrófila Mista no estado do Rio Grande do Sul, Brasil. Iheringia, Série Botânica 67: 47-57.

Koch NM, Branquinho C, Matos P, Pinho P, Lucheta F, Martins SMA \& Vargas VMF (2016) The application of lichens as ecological surrogates of air pollution in the subtropics: a case study in south Brazil. Environmental Science and Pollution Research 23: 20819-20834.

Kricke R \& Loppi S (2002) Bioindication: the I.A.P. aproach. In: Nimis L, Scheidegger C \& Wolseley P (eds.) Monitoring with lichens - monitoring lichens. Kluwer Academic Publishers, Dordrecht. Pp. 21-37.

Lättman H, Bergman K, Rapp M, Tälle M, Westerberg L \& Milberg P (2014) Decline in lichen biodiversity on oak trunks due to urbanization. Nordic Journal of Botany 32: 518-528.

Lücking R, Archer AW \& Aptroot A (2009) A world-wide key to the genus Graphis (Ostropales: Graphidaceae). The Lichenologist 41: 363-452.

Marcelli MP (1992) Ecologia liquênica nos manguezais do sul-sudeste brasileiro. Bibliotheca Lichenologica 47: 1-310.

Marcelli MP (1998) Hystory and current knowledge of Brazilian lichenology. In: Martins SMA, Käffer MI \& Lemos A(eds.) (2008) Liquens como bioindicadores da qualidade do ar numa área de termoelétrica, Rio Grande do Sul, Brasil. Hoenea 35: 425-433.

Martins SMA \& Marcelli MP (2011) Specific distribution of lichens on Dodonaea viscosa L. in the restinga area of Itapua State Park in Southern Brazil. Hoehnea 38: 397-411.

McCune B \& Mefford MJ (2011) PC-ORD. Multivariate analysis of ecological data, version 6.0 for Windows. Disponível em <https://www.pcord.com/>. Acesso em 10 novembro 2016.

McKinney ML (2006) Urbanization as a major cause of biotic homogenization. Biological Conservation 127: 247-260.

McKinney ML (2008) Effects of urbanization on species richness: a review of plants and animals. Urban Ecosystems 11: 161-176.

Munzi S, Correia O, Silva P, Lopes N, Freitas C, Branquinho C \& Pinho P (2014) Lichens as ecological indicators in urban areas: beyond the effects of pollutants. Journal of Applied Ecology 51: 1750-1757.

Nash III TH (2008) Introduction. In: Nash III TH (ed.) Lichen biology. University Press, Cambridge. Pp. 1-8.

Niemelä J \& Kotze DJ (2009) Carabid beetle assemblages along urban to rural gradients: a review. Landscape and Urban Planning 92: 65-71. 
Peel MC, Finlayson BL \& McMahon TA (2007) Updated world map of the Köppen-Geiger climate classification. Hydrology and Earth System Sciences 11: 1633-1644.

Pinho P, Moretti M, Luz AC, Grilo F, Vieira J, Luís L, Rosalino LM, Martins-Loução MA, Santos-Reis M, Correia O, Garcia-Pereira P, Gonçalves P, Matos P, Carvalho RC, Rebelo R, Dias T, Mexia T \& Branquinho C (2017) Biodiversity as support for ecosystem services and human wellbeing. In: Pearlmutter D, Calfapietra C, Samson R, O'Brien L, Krajter Ostoić S, Sanesi G \& Alonso del Amo R (eds.) The urban forest: cultivating green infrastructure for people and the environment. Springer International Publishing, Cham. Pp. 67-78.

Pinho P, Correia O, Lecoq M, Munzi S, Vasconcelos S, Gonçalves P, Rebelo R, Antunes C, Silva P, Freitas C, Lopes N, Santos-Reis M \& Branquinho C (2016) Evaluating green infrastructure in urban environments using a multi-taxa and functional diversity approach. Environmental Research 147: 601-610.

Rocha-Uriartt L, Cassanego MBB, Becker DFP, Droste A \& Schmitt JL (2015) Diagnóstico ambiental de mata ciliar: uma análise integrada de parâmetros botânicos, meteorológicos e da genotoxicidade do ar atmosférico. Revista Brasileira de Ciências Ambientais (online) 35: 102-115.

SEMA - Secretaria do Ambiente e Desenvolvimento Sustentável (2016) Bacia hidrográfica do Rio dos Sinos. Disponível em <http:/www.sema.rs.gov.br/ g020-bacia-hidrografica-do-rio-dos-sinos>. Acesso em 20 junho 2016.

Seto KC, Güneralp B \& Hutyra L (2012) Global forecasts of urban expansion to 2030 and direct impacts on biodiversity and carbon pools. PNAS 109: 16083-16088.

Spielmann AA \& Marcelli MP (2009) Parmotrema s.l. (Parmeliaceae, lichenized Ascomycota) from Serra Geral slopes in central Rio Grande do Sul state, Brazil. Hoehnea 36: 551-595.

Spribille T, Tuovinen V, Resl P, Vanderpool D, Wolinski H, Aime MC, Schneider K, Stabentheiner E, ToomeHeller M, Thor G, Mayrhofer H, Johannesson H \& McCutcheon JP (2016) Basidiomycete yeasts in the cortex of ascomycete macrolichens. Science 353: 488-492.

Washburn SJ \& Culley TM (2006) Epiphytic macrolichens of the greater Cincinnati metropolitan area - part II: distribution, diversity and urban ecology. The Bryologist 109: 516-526. 